BIORHEOLOGY Vo1. $16, \mathrm{p} .5$

Pergamon Press Ltd. 1979. Printed in Great Britain

\title{
THE COUNCIL OF THE INTERNATIONAL SOCIETY OF BIORHEOLOGY
}

\author{
$1978-1981$ \\ President \\ Past President \\ Vice Presidents \\ Secretary General \\ Treasurer \\ Council Members \\ Chairman, Committee \\ on Nomenclature \\ Eiichi Fukada, JAPAN \\ Biopolymer Physics Laboratory, Rikagaku Kenkyusho (The \\ Institute of Physical and Chemical Research), Wako- \\ shi, Saitama 351 \\ Alex Silberberg, ISRAEL \\ Polymer Department, The Weizmann Institute of Science, \\ Rehovot \\ Takehiko Azuma, JAPAN \\ Department of Physiology, Shinshu University Medical \\ School, Asahi Matsumoto, Nagano \\ Yuan-Cheng B. Fung, U.S.A. \\ Department of Applied Mechanics and Engineering Sci- \\ ences, M-005, University of California, San Diego, \\ La Jolla, CA 92037 \\ He11mut H. Hartert, F.R. GERMANY \\ Medizinische Klinik, Städtisches Krankenhaus \\ Friedrich-Engels-Strasse 25, 6750 Kaiserlautern \\ Vladimir I. Vorob'ev, U.S.S.R. \\ Institute of Cytology, Academy of Sciences, Lenin- \\ grad 190121 \\ Don E. Brooks, CANADA \\ Department of Pathology, University of British Col- \\ umbia, Vancouver V6T 1W5 \\ Siegfried Witte, F.R. GERMANY \\ Diakonissenkrankenhaus, Diakonissenstrasse 28, \\ 75 Karlsruhe 51 \\ Harry L. Goldsmith, CANADA \\ University Medical Clinic, The Montreal General Hos- \\ pital, Montreal H3G 1 A4 \\ Maurice Joly, FRANCE \\ Départment de Biophysique et de Biomathématiques, \\ Faculté de Médecine Pitié-Salpêtrière, Université \\ Paris 6, 91, Boulevard de Hôpital, 75634 Paris Cedex \\ 13. Or: 55 rue Lacordaire, 75015 Paris \\ Mitche11 Litt, U.S.A. \\ Department of Chemical and Biochemical Engineering, \\ University of Pennsylvania, Philadelphia, Pa. 19174 \\ Holger Schmid-Schönbein, F.R. GERMANY \\ Abteilung Physiologie, Medizinische Fakultät, Rhein.- \\ Westf. Technische Hochschule, Melatener Strasse 211 , \\ 51 Aachen \\ Alfred L. Copley, U.S.A. \\ Laboratory of Biorheology, Polytechnic Institute of \\ New York, 333 Jay Street, Brooklyn, N.Y. 11201. Or: \\ 50 Central Park West, New York, N.Y. 10023
}

Alex Silberberg, ISRAEL

Alfred L. Copley, U.S.A. 